\title{
COMPLEX LINDENSTRAUSS SPACES WITH EXTREME POINTS
}

\author{
BY
}

\author{
B. HIRSBERG AND A. J. LAZAR
}

\begin{abstract}
We prove that a complex Lindenstrauss space whose unit ball has at least one extreme point is isometric to the space of complex valued continuous affine functions on a Choquet simplex. If $X$ is a compact Hausdorff space and $A \subset C_{\mathrm{C}}(X)$ is a function space then $A$ is a Lindenstrauss space iff $A$ is selfadjoint and $\operatorname{Re} A$ is a real Lindenstrauss space.
\end{abstract}

1. In [3] and [4] E. Effros proposed and investigated the complex analogue of the preduals of real $L^{1}$-spaces, also called Lindenstrauss spaces. We aim to discuss those complex Lindenstrauss spaces whose unit balls have at least one extreme point. First, we prove a result which is well known in the case of real scalars (cf. [8]):

Theorem 1. Let $E$ be a Lindenstrauss space and $u$ an extreme point of the closed unit ball of $E$. Let $S=\left\{x^{*} \in E^{*}:\left\|x^{*}\right\|=x^{*}(u)=1\right\}$. For every $x \in E$ let $\hat{x} \in C_{C}(S)$ be defined by $\hat{x}\left(x^{*}\right)=x^{*}(x)$. Then $S$ is a $w^{*}$-compact subset of $E^{*}$ and the map $x \rightarrow \hat{x}$ of $E$ into $C_{C}(S)$ is an isometry sucb that $\hat{u}=1$ s.

More information about Lindenstrauss spaces as function spaces is given by

Theorem 2. Let $X$ be a compact Hausdorff space and $A \subset C_{C}(X)$ a closed linear subspace, separating the points of $X$ and containing the constant functions. Let $S$ denote the state space of $A$. Then the following statements are equivalent:

(i) $A$ is a Lindenstrauss space;

(ii) $\mu \in A^{\perp} \cap M\left(\partial_{A} X\right) \Rightarrow \mu=0$;

(iii) $Z=\operatorname{conv}(S U-i S)$ is a Choquet simplex;

(iv) $A$ is selfadjoint and $\operatorname{Re} A$ is a real Lindenstrauss space.

From Theorems 1 and 2 we shall get a characterization of $C_{C}(X)$ spaces identical to that given for real scalars in $\left[8, p_{0} 76\right]$ and we shall see that Theorem 2 implies that no uniform algebra is a Lindenstrauss space unless it is $C_{C}(X)$.

We shall follow the notations of [1]. By $l_{\infty}^{n}$ we shall denote the linear space of all sequences $a=\left(a_{1}, a_{2}, \ldots, a_{n}\right)$ of $n$ complex numbers with the norm $|a|$ $=\max _{1 \leq i \leq n}\left|a_{i}\right|$. If $E$ is a Banach space we shall denote its closed unit ball by $B(E)$.

Received by the editors November 28, 1972 and, in revised form, January 13, 1973. AMS (MOS) subject classifications (1970). Primary 46E 15.

Key words and phrases. Lindenstrauss space, maximal measure, Choquet simplex. 
The proof of Theorem 1 relies on results of [7] and [9]. The results and the proofs of [9] are clearly valid for complex scalars as well as for real scalars. Theorem 1 of [7] is proved for real scalars only and it is stated there that it is valid in the complex case too. For the reader's convenience we outline the needed changes. We have to prove that for every finite dimensional subspace $B$ of a Lindenstrauss space $X$ and every $\epsilon>0$ there is a natural number' $n$ and an operator $T: l_{\infty}^{n} \rightarrow X$ such that $(1-5 \epsilon)\|y\|<\|T y\|<(1+\epsilon)\|y\|$ for each $y \epsilon l_{\infty}^{n}$ and such that dist $\left(x, T l_{\infty}^{n}\right)<\epsilon$ for every $x \in B$ with $\|x\|=1$. Let $E_{0}$ be the set of all exposed points of the unit ball of $B^{*}$. Define in $E_{0}$ an equivalence relation by putting $f \sim g$ if $f=\theta g$ with $\theta \in C,|\theta|=1$. Let $\widetilde{E}_{0}$ be the quotient set and $\phi:$ $E_{0} \rightarrow \tilde{E}_{0}$ the quotient map. We metrize $\tilde{E}_{0}$ by the Hausdorff metric. Actually $d(\phi(f), \phi(g))=\min \left\{\left\|f_{1}-g_{1}\right\|: \phi\left(f_{1}\right)=\phi(f), \phi\left(g_{1}\right)=\phi(g)\right\}$. Clearly $\widetilde{E}_{0}$ is totally bounded. Thus there is a covering of $E_{0}$ consisting of mutually disjoint sets $\left\{\tilde{G}_{i}\right\}_{i=1}^{n}$ such that each $\tilde{G}_{i}$ has a nonvoid interior and is of diameter less than $\epsilon / 2$. Pick $\phi\left(g_{i}\right) \in \widetilde{G}_{i}$. It is easily seen that for every $\phi(g) \in \widetilde{G}_{i}$ there is $g^{\prime} \in E_{0}$ such that $\left\|g_{i}-g^{\prime}\right\|<\epsilon / 2$ and $\phi\left(g^{\prime}\right)=\phi(g)$. Thus for every $i$ there is a subset $G_{i}$ of $E_{0}$ such that $\phi\left(G_{i}\right)=\widetilde{G}_{i},\left.\phi\right|_{G_{i}}$ is one-to-one and $\|f-g\|<\epsilon$ if $f, g \in G_{i}$. From here on one may continue with very minor modifications as in [7].

2. An admissible basis in a space $E$ isometric to $l_{\infty}^{n}$ is the image of the unit vector basis of $l_{\infty}^{n}$ by an isometry of $l_{\infty}^{n}$ onto $E$.

Lemma 2.1. Let $E \subset F$ be Banach spaces such that $E$ is isometric to $l_{\infty}^{n}$ and $F$ is isometric to $l_{\infty}^{m}$. Let $P$ be a contractive projection of $F$ onto $E$ sucb that there is an admissible basis $\left\{e_{i}\right\}_{i=1}^{n}$ of $E$ and an admissible basis $\left\{u_{j}\right\}_{j=1}^{m}$ of $F$ for which $P\left(u_{j}\right)=e_{j}$ if $1 \leq j \leq n_{0} P\left(u_{j}\right)=0$ if $n+1 \leq j \leq m$. Let $x \in B(F)$ and assume that $P(x)=1 / 2(v+w), v, w \in B(E), v \neq P(x)$. Then there are $y, z \in B(F)$ such that $x=1 / 2(y+z), P(y)=v, P(z)=w$ and $\|y-v\|,\|z-w\| \leq$ $\max \left\{4\|x-P(x)\|, 4\|x-P(x)\|^{1 / 4}\right\}$.

Proof. From [9, Lemma 2.3] and the assumptions on $P$ it foll ows that there are scalars $\left\{a_{i j}\right\}_{1 \leq i \leq n}^{\dot{n}+1 \leq j \leq m}$ such that

$$
\begin{aligned}
& e_{i}=u_{i}+\sum_{j=n+1}^{m} a_{i j} u_{j}, \quad 1 \leq i \leq n, \\
& \sum_{i=1}^{n}\left|a_{i, j}\right| \leq 1, \quad n+1 \leq j \leq m .
\end{aligned}
$$

Let $x=\sum_{j=1}^{m} \lambda_{j} u_{j}$. Then $P(x)=\sum_{i=1}^{n} \lambda_{i} e_{i}, v=\sum_{i=1}^{n}\left(\lambda_{i}+\delta_{i}\right) e_{i}, w=$ $\sum_{i=1}^{n}\left(\lambda_{i}-\delta_{i}\right) e_{i}$ where 
(1)

$$
\max _{1 \leq j \leq m}\left|\lambda_{j}\right| \leq 1, \quad \max _{1 \leq i \leq n}\left|\lambda_{i} \pm \delta_{i}\right| \leq 1
$$

Denote $\epsilon=\|x-P(x)\|$. Since $P(x)=\sum_{i=1}^{n} \lambda_{i} u_{i}+\sum_{j=n+1}^{m}\left(\sum_{i=1}^{n} \lambda_{i} a_{i j}\right) u_{j}$, we have

$$
\left|\lambda_{j}-\sum_{i=1}^{n} \lambda_{1} a_{i j}\right| \leq \epsilon, \quad n+1 \leq j \leq m .
$$

Now, if $\epsilon>1 / 3$ put $y=\sum_{i=1}^{n}\left(\lambda_{i}+\delta_{i}\right) u_{i}+\sum_{j=n+1}^{m} \lambda_{j} u_{j}, z=\sum_{i=1}^{n}\left(\lambda_{i}-\delta_{i}\right) u_{i}$ $+\sum_{j=n+1}^{m} \lambda_{j} u_{j}$. Since from (1) it follows that $\delta_{i} \mid \leq 1$, we have

$$
\|y-v\|=\max _{n+1 \leq j \leq m}\left|\lambda_{j}-\sum_{i=1}^{n}\left(\lambda_{i}+\delta_{i}\right) a_{i j}\right| \leq \epsilon+1 \leq 4 \epsilon .
$$

Similarly, $\|z-w\| \leq 4 \epsilon$. Clearly $y$ and $z$ so defined satisfy all the required conditions.

Suppose now $0 \leq \epsilon<1 / 3$ and fix $j, n+1 \leq j \leq m$. If $\left|\lambda_{j}\right| \geq 1-\epsilon^{1 / 2}$ define $\delta_{j}=0$. If $\left|\lambda_{j}\right|<1-\epsilon^{1 / 2}$ and $\Sigma_{i=1}^{n} \delta_{i} a_{i j}=0$ define $\delta_{j}=0$ too. If $\left|\lambda_{j}\right|<1-\epsilon^{1 / 2}$ and $\sum_{i=1}^{n} \delta_{i} a_{i j} \neq 0$ define $\delta_{j}$ as follows. There are uniquely determined positive numbers $t_{1}, t_{2}$ such that

$$
\left|\lambda_{j}+t_{1} \sum_{i=1}^{n} \delta_{i} a_{i j}\right|=\left|\lambda_{j}-t_{2} \sum_{i=1}^{n} \delta_{i} a_{i j}\right|=1 .
$$

Put

$$
t=\min \left(1, t_{1}, t_{2}\right)
$$

$$
\delta_{j}=t \sum_{i=1}^{n} \delta_{i} a_{i j}
$$

For future use we remark that

$$
0 \leq 1-t \leq \epsilon^{1 / 2} \text {. }
$$

Indeed, if $t=1$ this is clear. If $t=t_{1}$ or $t=t_{2}$ then

$$
\begin{aligned}
1 & =\left|(1-t) \lambda_{j}+t\left(\lambda_{j} \pm \sum_{i=1}^{n} \delta_{i} a_{i j}\right)\right| \\
& \leq(1-t)\left|\lambda_{j}\right|+t\left|\lambda_{j} \pm \sum_{i=1}^{n} \delta_{i} a_{i j}\right| \leq(1-t)\left(1-\epsilon^{1 / 2}\right)+t(1+\epsilon) ;
\end{aligned}
$$

thus $t \geq\left(\epsilon^{1 / 2}+1\right)^{-1}$ and $1-t \leq \sqrt{\epsilon}$. In the proof we made use of $\left|\lambda_{j} \pm \sum_{i=1}^{n} \delta_{i} a_{i j}\right|$ $\leq 1+\epsilon$ which is a consequence of (1) and (2).

Define $y=\sum_{j=1}^{m}\left(\lambda_{j}+\delta_{j}\right) u_{j}, z=\sum_{j=1}^{m}\left(\lambda_{j}-\delta_{j}\right) u_{j}$. Clearly $x=1 / 2(y+z), P(y)$ $=v, P(z)=w$. For every $j$ we have $\left|\lambda_{j} \pm \delta_{j}\right| \leq 1$; thus $y, z \in B(F)$. Indeed, if $1 \leq j \leq n$ or if $n+1 \leq j \leq m$ and $\delta_{j}=0$ this is obvious. Otherwise this is a consequence of (3), (4) and (5). 
Now

Thus

$$
y-v=\sum_{j=n+1}^{m}\left[\lambda_{j}+\delta_{j}-\sum_{i=1}^{n}\left(\lambda_{i}+\delta_{i}\right) a_{i j}\right] u_{j}
$$

$$
\|y-v\|=\max _{n+1 \leq j \leq m}\left|\lambda_{j}+\delta_{j}-\sum_{i=1}^{n}\left(\lambda_{i}+\delta_{i}\right) a_{i j}\right| \text {. }
$$

Suppose $\left|\lambda_{j}\right| \geq 1-\epsilon^{1 / 2}$. Then $\left|\sum_{i=1}^{n} \lambda_{i} a_{i j}\right| \geq 1-\epsilon^{1 / 2}-\epsilon>0$ by (2) (recall that $0 \leq$ $\epsilon<1 / 3)$ and from this and $\left|\sum_{i=1}^{n}\left(\lambda_{i} \pm \delta_{i}\right) a_{i j}\right| \leq 1$ we get

Therefore

$$
\left|\sum_{i=1}^{n} \delta_{i} a_{i j}\right| \leq\left(1-\left|\sum_{i=1}^{n} \lambda_{i} a_{i j}\right|^{2}\right)^{1 / 2} \leq 2 \epsilon^{1 / 4} .
$$

$$
\begin{gathered}
\left|\lambda_{j}+\delta_{j}-\sum_{i=1}^{n}\left(\lambda_{i}+\delta_{i}\right) a_{i j}\right| \leq\left|\lambda_{j}-\sum_{i=1}^{n} \lambda_{i} a_{i j}\right|+\left|\sum_{i=1}^{n} \delta_{i} a_{i j}\right| \\
\leq \epsilon+2 \epsilon^{1 / 4} \leq 4 \epsilon^{1 / 4} .
\end{gathered}
$$

If $\left|\lambda_{j}\right|<1-\epsilon^{1 / 2}$ and $\Sigma_{i=1}^{n} \delta_{i} a_{i j}=0$ then obviously $\left|\lambda_{j}+\delta_{j}-\sum_{i=1}^{n}\left(\lambda_{i}+\delta_{i}\right) a_{i j}\right| \leq$ $4 \epsilon^{1 / 4}$. Suppose now $\left|\lambda_{j}\right|<1-\epsilon^{1 / 2}$ and $\sum_{i=1}^{n} \delta_{i} a_{i j} \neq 0$. Then

$$
\begin{aligned}
\mid \lambda_{j}+\delta_{j} & -\sum_{i=1}^{n}\left(\lambda_{i}+\delta_{i}\right) a_{i j} \mid \\
& \leq\left|\lambda_{j}-\sum_{i=1}^{n} \lambda_{i} a_{i j}\right|+(1-t)\left|\sum_{i=1}^{n} \delta_{i} a_{i j}\right| \leq \epsilon+\epsilon^{1 / 2} \leq 4 \epsilon^{1 / 4}
\end{aligned}
$$

by (1), (2) and (6). It follows from this discussion that $\|y-v\| \leq 4 \epsilon^{1 / 4}$ and in the same way one can show that $\|z-w\| \leq 4 \epsilon^{1 / 4}$. The proof of the lemma is complete.

Let $E$ be a separable infinite dimensional Lindenstrauss space. Then there is an increasing sequence of subspaces $\left\{E_{n}\right\}_{n=1}^{\infty}$ such that $E=\overline{\bigcup_{n=1}^{\infty} E_{n}}$ and each $E_{n}$ is isometric to $l_{\infty}^{n}$ (cf. [7]). By [9, Lemma 2.3] one may choose admissible bases $\left\{e_{n}^{i}\right\}_{i=1}^{n}$ in every $E_{n}$ such that $e_{n}^{i}=e_{n+1}^{i}+a_{n}^{i} e_{n+1}^{n+1}, 1 \leq i \leq n$, and $\sum_{i=1}^{n}\left|a_{n}^{i}\right| \leq 1$ for $n=1,2, \ldots$. As in [10] we define a sequence of functionals $\left\{\phi_{i}\right\}_{i=1}^{\infty}$ on $\bigcup_{i=1}^{\infty} E_{i}$ as follows: for $i \leq n$ and $x=\sum_{i=1}^{n} \alpha_{i} e_{n}^{i}$ let $\phi_{i}(x)=\alpha_{i}$. Clearly $\phi_{i}$ is a well-defined linear functional and $\left\|\phi_{i}\right\|=1$. Thus $\phi_{i}$ can be extended by continuity to all of $E$.

Lemma 2. Let $E$ be a separable Lindenstrauss space and let $u \in \partial_{e} B(E)$. Suppose $E=\bigcup_{n=1}^{\infty} E_{n}$ where $\left\{E_{n}\right\}_{n=1}^{\infty}$ is an increasing sequence of subspaces sucb that $E_{n}$ is isometric to $l_{\infty}^{n}$ for every $n$. Then there is a sequence of contractive projections $\left\{P_{n}\right\}_{n=1}^{\infty}$ such that $\lim _{n \rightarrow \infty} P_{n}(x)=x$ for all $x \in E, P_{n}(E)=$ $E_{n}$ and $P_{n}(u) \in \partial_{e} B\left(E_{n}^{n}\right), n=1,2, \ldots$. 
Proof. Let $P_{n}(x)=\Sigma_{i=1}^{n} \phi_{i}(x) e_{n}^{i}$ where $e_{n}^{i}, \phi_{i}$ are defined as above. We have to show only that $P_{n}(u) \in \partial_{e} B\left(E_{n}\right)$. Assume that for some $n_{0^{\prime}} P_{n_{0}}(u)$ is not an extreme point of $B\left(E_{n_{0}}\right)$. Then $P_{n_{0}}(u)=1 / 2\left(x_{0}+y_{0}\right)$ with $x_{0} y_{0} \in B\left(E_{n_{0}}\right), x_{0} \neq y_{0}$. Choose an increasing sequence of natural numbers $\left\{n_{k}\right\}_{k=1}^{\infty}$ such that $n_{1}>n_{0}$ and $\left\|P_{n_{k+1}}(u)-P_{n_{k}}(u)\right\|<2^{-4 k}$. Since for any $m, n$ with $m \geq n$ we have $P_{n} P_{m}=P_{n}$ it follows from Lemma 2.1 that there are $x_{1}, y_{1} \in B\left(E_{n_{1}}\right)$ such that $P_{n_{1}}(u)=$ $1 / 2\left(x_{1}+y_{1}\right), P_{n_{0}}\left(x_{1}\right)=x_{0}, P_{n_{0}}\left(y_{1}\right)=y_{0^{\circ}}$. By using once more Lemma 1 we may construct inductively two sequences $\left\{x_{k}\right\}_{k=1}^{\infty},\left\{y_{k}\right\}_{k=1}^{\infty}$ such that for any $k$ we have $x_{k^{\prime}} y_{k} \in B\left(E_{n_{k}}\right), P_{n_{k}}(u)=1 / 2\left(x_{k}+y_{k}\right), P_{n_{k}}\left(x_{k+1}\right)=x_{k}, P_{n_{k}}\left(y_{k+1}\right)=y_{k}$ and $\left\|y_{k+1}-y_{k}\right\|,{ }^{n}\left\|x_{k+1}-x_{k}\right\| \leq 4 \cdot 2^{-k}$. Thus $\left\{x_{k}\right\}_{k=1}^{\infty},\left\{y_{k}\right\}_{k=1}^{\infty}$ converge to $x, y \in B(E)$ respectively and $u=1 / 2(x+y)$. Obviously $\|u-x\| \geq\left\|P_{n_{0}}(u)-x_{0}\right\|>0$ and this is a contradiction.

Lemma 2.3. Let $E$ be a Lindenstrauss space and $u \in \partial_{e} B(E)$. Then for any $x \in E,\|x\|=1$ and for any $\epsilon>0$ there is a functional $x^{*} \in E^{*}$ sucb that $\left\|x^{*}\right\|=$ $x^{*}(u)=1$ and $\left|x^{*}(x)\right|>1-\epsilon$.

Proof. By the proof of Theorem 1.1 in [9] there is a separable Lindenstrauss subspace $Y$ of $E$ such that $u, x \in Y$. Let $Y={\overline{U_{n=1}^{\infty} E_{n}}}_{\text {where }}\left\{E_{n}\right\}_{n=1}^{\infty}$ is an increasing sequence of finite dimensional subspaces such that $E_{n}$ is isometric to $l_{\infty}^{n}$ and let $\left\{P_{n}\right\}_{n=1}^{\infty}$ be a sequence of projections as given by Lemma 2.2. Choose $n$ such that $\left\|x-P_{n}(x)\right\|<\epsilon$. Since $P_{n}(u) \in \partial_{e} B\left(E_{n}\right)$ there is an $i, 1 \leq i \leq n$, for which $\left|\phi_{i}\left(P_{n}(x)\right)\right|>1-\epsilon$ and $\left|\phi_{i}\left(P_{n}(u)\right)\right|=1, \phi_{i}$ being one of the functionals on $Y$ defined in the discussion preceding Lemma 2.2. Now, $\phi_{i}(x)=\phi_{i}\left(P_{n}(x)\right)$ and $\phi_{i}(u)=\phi_{i}\left(P_{n}(u)\right)$. To get the desired functional one has to multiply $\phi_{i}$ by an appropriate constant $\theta$ and to extend $\theta \phi_{i}$ to $E$ by the Hahn-Banach theorem.

Proof of Theorem 1. Obviously $\|\hat{x}\| \leq\|x\|$ and by Lemma 2.3 we have $\|\hat{x}\| \geq$ $\|x\|$ for every $x \in E$.

3. Let $X$ be a compact Hausdorff space and let $A \subset C_{C}(X)$ be a closed linear subspace, separating the points of $X$ and containing the constant functions. For simplicity we shall denote $B\left(A^{*}\right)$ by $K$. The state space of $A$, i.e.

$$
S=\left\{p \in A^{*}: p\left(1_{x}\right)=\|p\|=1\right\}
$$

is a $w^{*}$-closed face of $K$. Define $Z=\operatorname{conv}(S U-i S)$ and let $\theta: A \rightarrow A(Z)$ be defined as $\theta a(z)=\operatorname{Re} z(a)$ for all $z \in Z$ and $a \in A$. Then $\theta$ is a bicontinuous reallinear isomorphism of $A$ onto the space $A(Z)$ of real continuous affine functions on $Z$ (cf. [2]).

We note that $S$ is a closed face of $Z$ with complementary face $S^{\prime}=-i S$. Moreover, the barycenter coefficient in the decomposition after $S$ and $S^{0}$ is uniquely determined, i.e. $S$ is a parallel face of $Z$. For details we refer to [1]. 
Let $\phi$ denote the canonical embedding of $X$ into $S$, i.e.

$$
\phi(x)(a)=a(x), \quad \forall a \in A .
$$

Also let $T$ denote the unit circle and define $\phi: T \times X \rightarrow K$ by $\Phi(\lambda, x)=\lambda \phi(x)$ and $L: C_{C}(X) \rightarrow C_{C}(T \times X)$ by

$$
L f(\lambda, x)=\lambda f(x), \quad \forall(\lambda, x) \in T \times X .
$$

It follows from [5] and [6] that $L^{*} \circ \Phi^{-1}$ maps maximal probability measures on $K$ into complex boundary measures on $X$, i.e. $\mu \in M_{1}^{+}\left(\partial_{e} K\right)$ implies $L^{*}\left(\Phi^{-1} \mu\right)$ $\epsilon M\left(\partial_{A} X\right)$.

Following Effros [4] we define for $f \in C_{C}(K)$ the function

$$
\operatorname{inv}_{T} f(p)=\int_{T} f(\alpha p) d \alpha, \quad \forall p \in K,
$$

where $d a$ is the normalized Haar measure on $T$. It is easily verified that in ${ }_{T}$ is a norm decreasing projection in $C_{\mathrm{C}}(K)$. Similarly, we write

$$
\operatorname{hom}_{T} f(p)=\int_{T} a^{-1} f(a p) d a, \quad \forall p \in K,
$$

and observe that hom $T$ is also a contractive projection in $C_{C}(K)$. Taking adjoints of these projections we obtain the following contractive $w^{*}$-continuous projections in $M(K)$ :

$$
\operatorname{inv}_{T} \mu=\mu \circ \text { inv }_{T}, \quad \operatorname{hom}_{T} \mu=\mu \circ \operatorname{hom}_{T} .
$$

In [4] Effros proved that a complex Lindenstrauss space $V$ can be characterized by the following condition on $B\left(V^{*}\right)$ :

(*) $\mu, \nu \in M_{1}^{+}\left(\partial_{e} B\left(V^{*}\right)\right)$ with $r(\mu)=r(\nu) \Rightarrow$ hom $_{T} \mu=\operatorname{hom}_{T} \nu$.

Here $r: M_{1}^{+}\left(B\left(V^{*}\right)\right) \rightarrow B\left(V^{*}\right)$ denotes the barycentric map.

Lemma 3.1. Let $\mu \in M_{1}^{+}\left(\partial_{e} K\right)$. Then the measures $\operatorname{hom}_{T}^{\mu}$ and $\operatorname{inv}_{T} \mu$ are boundary measures on $K$.

Proof. [4, Lemma 4.2].

If $\nu \in M(X)$, then we denote by $\phi(\nu)$ the direct image of $\nu$ under $\phi$.

Lemma 3.2. Let $\mu \in M_{1}^{+}\left(\partial_{e} K\right)$. Then the measure $\nu=L^{*}\left(\Phi^{-1} \mu\right)$ is a complex boundary measure on $X$ such that $\operatorname{hom}_{T} \mu=\operatorname{hom}_{T}(\phi \nu)$.

Proof. By [5] $\nu$ is a complex boundary measure on $X$.

Let $f \in C_{\mathrm{C}}(K)$. Then

$$
L\left(\operatorname{hom}_{T} f \circ \phi\right)(\lambda, x)=\operatorname{hom}_{T} f(\lambda \phi(x))
$$


Hence

$$
\begin{aligned}
\operatorname{hom}_{T}(\phi \nu)(f) & =\int_{X} \operatorname{hom}_{T} f \circ \phi d \nu=\int_{X} \operatorname{hom}_{T} f \circ \phi d L^{*}\left(\Phi^{-1} \mu\right) \\
& =\int_{T \times X} L\left(\operatorname{hom}_{T} f \circ \phi\right) d \Phi^{-1} \mu=\int_{\Phi(T \times X)}\left(L\left(\operatorname{hom}_{T} f \circ \phi\right)\right) \circ \Phi^{-1} d \mu \\
& =\int_{K} \operatorname{hom}_{T} f d \mu=\operatorname{hom}_{T} \mu(f)
\end{aligned}
$$

and the lemma is proved.

We shall need the following fact on the embedding of $S$ in $Z$.

Lemma 3.3. $S$ is a split face of $Z$ if and only if $A$ is closed under complex conjugation.

Proof. Assume $S$ is a split face of $Z$. Let $a \in A$ and decompose $a=$ $a_{1}+i a_{2}$.

Define $b_{1} \in A(S)$ and $b_{2} \in A(-i S)$ by

$$
b_{1}(p)=\theta a(p), \quad b_{2}(-i p)=-\theta a(-i p), \quad \forall p \in S .
$$

Since $S$ is a split face of $Z$ and $S^{\prime}=i S$ is closed, it follows from [1, Proposition II. 6.19] that there exists $b \in A$ such that $\left.\theta b\right|_{S}=b_{1},\left.\theta b\right|_{s^{\prime}}=b_{2}$. Thus for $x \in X$ we shall have $a_{1}(x)-i a_{2}(x)=\theta a(x)-i \theta a(-i x)=\theta b(x)+i \theta b(-i x)=b(x)$. Hence $\bar{a} \in A$.

Conversely, we assume that $A$ is closed under complex conjugation. Consider convex combinations

$$
\lambda p_{1}+(1-\lambda)\left(-i q_{1}\right)=\lambda p_{2}+(1-\lambda)\left(-i q_{2}\right)
$$

where $p_{i}, q_{i} \in S$ and $0<\lambda<1$ for $i=1,2$.

If $p_{1} \neq p_{2}$, then it follows from the Hahn-Banach theorem and the assumption on $A$ that we can find $a=\bar{a} \in A$ such that $\theta(a)\left(p_{1}\right) \neq \theta(a)\left(p_{2}\right)$. Moreover, $\lambda \theta a\left(p_{1}\right)+(1-\lambda) \theta a\left(-i q_{1}\right)=\lambda \theta a\left(p_{2}\right)+(1-\lambda) \theta a\left(-i q_{2}\right)$. Since $\left.\theta a\right|_{s^{\prime}}=0$, we shall have $\lambda \theta a\left(p_{1}\right)=\lambda \theta a\left(p_{2}\right)$ which is a contradiction, and the lemma is proved.

Proof of Theorem 2. (i) $\Rightarrow$ (ii). Let $\mu \in A^{\perp} \cap M\left(\partial_{A} X\right)$ and decompose $\mu$ as

$$
\mu=\lambda_{1} \mu_{1}-\lambda_{2} \mu_{2}+i \lambda_{3} \mu_{3}-i \lambda_{4} \mu_{4},
$$

where $\lambda_{i} \geq 0$ and $\mu_{i} \in M_{1}^{+}\left(\partial_{A} X\right)$ for $i=1,2,3,4$.

Let $p_{i}=r\left(\phi\left(\mu_{i}\right)\right)$ for $i=1,2,3,4$. Then

$$
0=\lambda_{1} p_{1}-\lambda_{2} p_{2}+i \lambda_{3} p_{3}-i \lambda_{4} p_{4},
$$

or equivalently 


$$
\lambda_{1} p_{1}+\lambda_{4}\left(-i p_{4}\right)=\lambda_{2} p_{2}+\lambda_{3}\left(-i p_{3}\right)
$$

Let $z$ be the common value of the left- and right-hand sides of this equation.

Since $1_{X} \in A$ we conclude that $\lambda_{1}=\lambda_{2}$ and $\lambda_{3}=\lambda_{4}$. Hence we may assume that $\lambda_{1}+\lambda_{4}=\lambda_{2}+\lambda_{3}=1$. Specifically, $z \in Z$.

If $\psi: K \rightarrow K$ is defined by $\psi(p)=-i p, \forall p \in K$, then the measures

$$
\nu_{1}=\lambda_{1}\left(\phi\left(\mu_{1}\right)\right)+\lambda_{4} \psi\left(\phi\left(\mu_{4}\right)\right), \quad \nu_{2}=\lambda_{2}\left(\phi\left(\mu_{2}\right)\right)+\lambda_{3} \psi\left(\phi\left(\mu_{3}\right)\right),
$$

are maximal probability measures representing $z$.

Since $A$ is a complex Lindenstrauss space, it satisfies the condition (*). Hence $\operatorname{hom}_{T} \nu_{1}=\operatorname{hom}_{T} \nu_{2}$.

Let $f \in C_{\mathrm{C}}(X)$; define $\bar{f}$ on $\Phi(T \times X)$ by

$$
\bar{f}(\lambda \phi(x))=\lambda f(x)
$$

and extend $\bar{f}$ to $\bar{f} \in C_{\mathrm{C}}(K)$ (Tietze). Then

$$
\operatorname{hom}_{T} \bar{f}(\phi(x))=f(x), \quad \forall x \in X .
$$

Moreover,

Similarly,

$$
\begin{aligned}
\operatorname{hom}_{T} \nu_{1}(\bar{f}) & =\lambda_{1} \int_{X} \operatorname{hom}_{T} \bar{f} \circ \phi d \mu_{1}+\lambda_{4} \int_{X} \operatorname{hom}_{T} \bar{f} \circ \psi \circ \phi d \mu_{4} \\
& =\lambda_{1} \int_{X} f d \mu_{1}-i \lambda_{4} \int_{X} f d \mu_{4} .
\end{aligned}
$$

$$
\operatorname{hom}_{T^{\nu}} \nu_{2}(\bar{f})=\lambda_{2} \int_{X} f d \mu_{2}-i \lambda_{3} \int_{X} f d \mu_{3},
$$

and hence

$$
0=\lambda_{1} \mu_{1}(f)-\lambda_{2} \mu_{2}(f)+i \lambda_{3} \mu_{3}(f)-i \lambda_{4} \mu_{4}(f)=\mu(f),
$$

i.e. $\mu=0$ and (ii) follows.

(ii) $\Rightarrow$ (i). The condition $(*)$ is seen to be an immediate consequence of Lemma 3.2.

(ii) $\Rightarrow$ (iii). First we observe that $S$ is a Choquet simplex since (ii) asserts that there are no real annihilating boundary measures. Hence it suffices to prove that $S$ is a split face of $Z$ or equivalently that $A$ is selfadjoint.

To see this we assume that $a \in A$ and $\bar{a} \notin A$. Then there exists a measure $\mu \in A^{\perp}$ such that $\mu(\bar{a}) \neq 0$. Decompose $\mu$ into real and imaginary parts, i.e. $\mu=\mu_{1}+i \mu_{2}$ and choose real boundary measures $\nu_{i} \in M\left(\partial_{A} X\right)$ such that

$$
\mu_{i}-\nu_{i} \in A^{\perp} \quad \text { for } i=1,2
$$


Define $\nu=\nu_{1}+i \nu_{2}$; then $\nu \in A^{\perp} \cap M\left(\partial_{A} X\right)$ and from (ii) we conclude that $\nu=0$ and hence $\nu_{1}=0=\nu_{2}$. In particular $\mu_{i} \in A^{\perp}$ for $i=1,2$ and hence $\mu(\bar{a})=0$, and we have obtained a contradiction.

(iii) $\Rightarrow$ (ii). Let $\mu \in A^{\perp} \cap M\left(\partial_{A} X\right)$ and decompose $\mu$ as $\mu=\mu_{1}+i \mu_{2}$. Since $A$ is selfadjoint, we have that $\mu_{i} \in A^{\perp}$ for $i=1,2$, and since $S$ is a Choquet simplex, we conclude that $\mu_{i}=0$ for $i=1,2$ and hence $\mu=0$.

(iii) $\Leftrightarrow$ (iv). Trivial.

This completes the proof of the theorem.

Remark. In order to prove that (ii) implies (i) we could have used the fact that the space of complex boundary measures $M\left(\partial_{A} X\right)$ is an $L^{1}$-space [4], and since every $p \in A^{*}$ can be represented by a complex boundary measure $\mu_{p} \epsilon$ $M\left(\partial_{A} X\right)$ with $\|p\|=\left\|\mu_{p}\right\|$, condition (ii) asserts that $A^{*}$ is isometrically isomorphic to $M\left(\partial_{A} X\right)$, and (i) follows.

Corollary 3.4. Let $E$ be a complex Banach space. $E$ is isometric to a $C_{\mathrm{C}}(X)$ space iff it satisfies the following conditions

(i) $E$ is a Lindenstrauss space;

(ii) $\partial_{e} B\left(E^{*}\right)$ is $w^{*}$ closed;

(iii) $\partial_{e} B(E) \neq \varnothing$.

Proof. If $E$ is a $C_{\mathrm{C}}(X)$ space then (i)-(iii) are well known. Assume that $E$ fulfills these conditions and let $u \in \partial_{e} B(E)$. By Theorems 1 and $2, E$ is isometric to the space of complex continuous affine functions on $S=\left\{x^{*} \in E^{*}: x^{*}(u)=\right.$ $\left.\left\|x^{*}\right\|=1\right\}$ in the $w^{*}$-topology. By (ii) and Theorem 2, $S$ is a Bauer simplex; hence $E$ is isometric to $C_{c}\left(\partial_{e} S\right)$ (cf. [1, Theorem II. 4.3]).

The real analogue of the above corollary was proved by Lindenstrauss $[8$, p. 76].

Corollary 3.5. Let $A$ be a uniform subalgebra of $C_{C}(X)$. Then $A$ is a Lindenstrauss space iff $A=C_{C}(X)$.

Proof. If $A$ is a Lindenstrauss space then $A$ is selfadjoint. The StoneWeierstrass theorem yields $A=C_{\mathrm{C}}(X)$.

\section{REFERENCES}

1. E. M. Alfsen, Compact convex sets and boundary integrals, Ergebnisse der Mathematik und ihrer Grenzgebiete, Heft 57, Springer-Verlag, Berlin and New York, 1971.

2. L. Asimow, Decomposable compact convex sets and peak sets for function spaces, Proc. Amer. Math. Soc. 25 (1970), 75-79. MR 41 \#4244.

3. E. Effros, On a class of real Banach spaces, Israel J. Math. 9 (1971), 430-458.

4. - On a class of complex Banach spaces (to appear). 
5. B. Hirsberg, Représentations intégrales des formes linéaires complexes, C. R. Acad. Sci. Paris Sér. A-B 274 (1972), A1222.

6. O. Hustad, A norm-preserving complex Choquet theorem, Math. Scand. 29 (1971), 272-278.

7. A. J. Lazar and J. Lindenstrauss, On Banach spaces whose duals are $L_{1}$-spaces, Israel J. Math. 4 (1966), 205-207. MR 34 \#6488.

8. J. Lindenstrauss, Extension of compact operators, Mem. Amer. Math. Soc. No. 48 (1964). MR 31 \#3828.

9. E. Michael and A. Pekczyński, Separable Banach spaces which admit $l_{n}^{\infty}$ approximations, Israel J. Math. 4 (1966), 189-198. MR 35 \#2129.

10. M. Zippin, On some subspaces of Banach spaces whose duals are $L_{1}$ spaces, Proc. Amer. Math. Soc. 23 (1969), 378-385. MR 39 \#400.

INSTITUTE OF MATHEM ATICS, AARHUS UNIVERSITY, AARHUS, DENMARK

DEPARTMENT OF MATHEMATICS, TEL-AVIV UNIVERSITY, TEL-AVIV, ISRAEL 